Pacific Journal of Mathematics

SYMMETRIC MAXIMAL IDEALS IN $M(G)$ 


\section{SYMMETRIC MAXIMAL IDEALS IN $M(G)$}

\section{SADAHIRO SAEKI}

Let $G$ be a nondiscrete locally compact abelian group, and $M(G)$ the convolution algebra of bounded regular measures on $G$. In this paper, the following is proved: Let $\left\{\lambda_{k}\right\}_{k=0}^{\infty}$ be a countable subset of $M_{c}^{+}(G), 0 \neq \lambda_{0} \in M_{0}(G)$, and $\left\{C_{k}\right\}_{k=0}^{\infty}$ a countable family of $\sigma$-compact subsets of $G$ such that $\lambda_{k}\left(x+C_{k}\right)=0$ for all $x \in G$ and all $k=0,1,2, \cdots$. Then there exists a nonzero measure $\sigma \in M_{0}^{+}\left(\operatorname{supp} \lambda_{0}\right)$ with compact support such that $\lambda_{k}\left[x+C_{k}+G_{p}(\operatorname{supp} \sigma)\right]=0$ for all $x \in G$ and all $k=$ $0,1,2, \cdots$. A consequence of this result is the following: Let $Y$ be the closed ideal in $M(G)$ which is generated by U $\left\{L^{1}\left(\lambda_{k}\right): k=0,1,2, \cdots\right\}$ for some countable subset $\left\{\lambda_{k}\right\}_{h=0}^{\infty}$ of $M_{c}(G)$. Then there exist "fairly many" symmetric maximal ideals in $M(G)$ which contain $\cup\left\{L^{1}(\mu): \mu \in Y\right\} \cup M_{a}(G)$ but not $M_{0}(G)$. Here $L^{1}(\mu)$ denotes the set of the measures in $M(G)$ which are absolutely continuous with respect to $|\mu|$.

Throughout the paper, let $G$ be a nondiscrete locally compact abelian group, $\hat{G}$ its dual, and $M(G)$ the convolution algebra of bounded regular measures on $G$. We use the following customary notations:

$$
L^{1}(G)=M_{a}(G) \subset M_{0}(G) \subset M_{c}(G) \subset M(G) .
$$

Here $M_{0}(G)$ denotes the closed ideal of those measures in $M(G)$ whose Fourier transforms vanish at infinity. For the definitions of $M_{a}(G)$ and $M_{c}(G)$, see [3:(19.13)]; for the second inclusion, see [8:5.6.9] or [4]. Given a measure $\mu \in M(G)$, we denote by $L^{1}(\mu)$ the set of those measures in $M(G)$ which are absolutely continuous with respect to $|\mu|$. For a set $K$ in $G$, define

$$
(K)_{1}=K \cup(-K) \text { and }(K)_{n}=(K)_{n-1}+(K)_{1} \quad(n=2,3, \cdots) .
$$

Thus, the union of all $(K)_{n}$, denoted by $G_{p}(K)$, is the subgroup of $G$ generated by $K$.

Our main results are the following.

THEOREM 1. Let $\left\{\lambda_{k}\right\}_{k=0}^{\infty}$ be a countable subset of $M_{c}^{+}(G), 0 \neq \lambda_{0} \in$ $M_{0}(G)$, and $\left\{C_{k}\right\}_{k=0}^{\infty}$ a countable family of nonempty $\sigma$-compact subsets of $G$ such that

$$
\lambda_{k}\left(x+C_{k}\right)=0 \quad(x \in G ; k=0,1,2, \cdots) .
$$

Then there exists a nonzero measure $\sigma \in M_{0}^{+}\left(\operatorname{supp} \lambda_{0}\right)$ with compact support such that 
(b)

$$
\lambda_{k}\left[x+C_{k}+G_{p}(\operatorname{supp} \sigma)\right]=0 \quad(x \in G ; k=0,1,2, \cdots) .
$$

If, in addition, $G$ is metrizable, such a measure $\sigma$ can be taken so that $(\operatorname{supp} \sigma)-x_{0}$ is independent for some $x_{0} \in G$.

CoROLlary. Let $\left\{\lambda_{k}\right\}_{k=0}^{\infty}$ be a countable subset of $M_{c}(G), 0 \neq \lambda_{0} \epsilon$ $M_{0}(G)$, and $Y$ the closed ideal in $M(G)$ which is generated by $\mathrm{U}\left\{L^{1}\left(\lambda_{k}\right): k=0,1,2, \cdots\right\}$. Then there exists a nonzero measure $\sigma \in M_{0}^{+}\left(\operatorname{supp} \lambda_{0}\right)$ with compact support such that

$$
|\mu|\left[x+G_{p}(\operatorname{supp} \sigma)\right]=0 \quad(x \in G ; \mu \in Y) .
$$

If, in addition, $G$ is metrizable, such a measure $\sigma$ can be taken so that $(\operatorname{supp} \sigma)-x_{0}$ is independent for some $x_{0} \in G$.

Theorem 2. Let $\left\{\lambda_{k}\right\}_{k=0}^{\infty}$ and $Y$ be as in the Corollary. Then there exists a symmetric maximal ideal $\Theta$ in $M(G)$ such that

$$
\bigcup\left\{L^{1}(\mu): \mu \in Y\right\} \cup M_{a}(G) \subset \Theta \quad \text { but } M_{0}(G) \not \subset \Theta .
$$

Furthermore, the set of all $\Theta$ 's with these properties has cardinal number larger than or equal to $\max \left\{2^{\omega}\right.$, Card $\left.\hat{G}\right\}$. Here $\omega$ denotes the smallest uncountable cardinal.

Theorem 1 improves the main result in [6] and its Corollary generalizes a theorem of Rudin [7] (see N. Th. Varopoulos [9] in this connection). The idea of our proof is due to T. W. Körner [5: Ch. XIII]. Although the arguments needed are similar to those in [6], we give a detailed proof of Theorem 1 .

We need some lemmas.

LEMma 1. Let $\lambda$ be a measure in $M_{c}^{+}(G)$, and $D$ a compact subset of $G$ such that $\lambda(x+D)=0$ for all $x \in G$. Then, for each finite set $F$ in $G, n \in N$ (the natural numbers) and $\varepsilon>0$, there exists a neighborhood $V$ of $0 \in G$ such that

$$
\lambda\left[x+D+(F+V)_{n}\right]<\varepsilon \quad(x \in G) .
$$

Proof. Let $F, n$, and $\varepsilon$ be as above. Take a compact set $K$ in $G$ so that $\lambda(G \backslash K)<\varepsilon$, and fix any neighborhood $V_{0}$ of 0 with compact closure. Since $F$ is finite, $\lambda\left[x+D+(F)_{n}\right]=0$ for all $x \in G$ by hypothesis. Thus, for each $x \in G$, we can find a neighborhood $W_{x}$ of 0 so that

$$
\lambda\left[x+D+(F)_{n}+\left(W_{x}\right)_{2 n}\right]<\varepsilon .
$$

(Note that $D+(F)_{n}$ is compact.) It follows from compactness of the 
set $K-\left[D+(F)_{n}+\left(\bar{V}_{0}\right)_{n}\right]$ that there exist finitely many points $x_{1}$, $x_{2}, \cdots, x_{m} \in G$ such that

$$
K-\left[D+(F)_{n}+\left(V_{0}\right)_{n}\right] \subset \bigcup_{j=1}^{m}\left(x_{j}+W_{x_{j}}\right) .
$$

Put $V=\bigcap_{j=1}^{m} W_{x_{j}} \cap V_{0}$. If $x \in K-\left[D+(F)_{n}+\left(V_{0}\right)_{n}\right]$, then $x \in x_{j}+$ $W_{x_{j}}$ for some $j=j(x)$, and so

$$
\begin{aligned}
\lambda\left[x+D+(F+V)_{n}\right] & \leqq \lambda\left[x_{j}+W_{x_{j}}+D+(F)_{n}+(V)_{n}\right] \\
& \leqq \lambda\left[x_{j}+D+(F)_{n}+\left(W_{x_{j}}\right)_{2 n}\right]<\varepsilon .
\end{aligned}
$$

If $x \notin K-\left[D+(F)_{n}+\left(V_{0}\right)_{n}\right]$, then

$$
\left[x+D+(F+V)_{n}\right] \cap K \subset\left[x+D+(F)_{n}+\left(V_{0}\right)_{n}\right] \cap K=\varnothing,
$$

and so $\lambda\left[x+D+(F+V)_{n}\right] \leqq \lambda(G \backslash K)<\varepsilon$. This completes the proof.

LeMma 2. Suppose that $G$ is metrizable and $\lambda_{0}$ a nonzero measure in $M_{c}^{+}(G)$. Then there exists a point $x_{0} \in G$ and a nonempty, totally disconnected, compact, perfect subset $K_{0}$ of supp $\lambda_{0}$ with the following three properties.

(a) Every nonempty (relatively) open subset of $K_{0}$ has positive $\lambda_{0}$-measure.

(b) The elements of $K_{0}-x_{0}$ have the same order, say $q_{0}$.

(c) If $V_{1}, V_{2}, \cdots, V_{m}$ are $m$ disjoint, nonempty, open subsets of $K_{0}$, there exist $m$ points $x_{j} \in V_{j}$ such that $x_{1}-x_{0}, x_{2}-x_{0}, \cdots, x_{m}-x_{0}$ are independent.

Proof. Since $G$ is metrizable and $\lambda_{0}$ is continuous, we may assume that $\lambda_{0}$ is carried by a totally disconnected compact set.

Suppose first that there exist a natural number $q$ and an element $y \in G$ such that

$$
E(q, y)=\left\{x \in \operatorname{supp} \lambda_{0}: q x=y\right\}
$$

has positive $\lambda_{0}$-measure. Let $q_{0}$ be the smallest natural number such that $\lambda_{0}\left[E\left(q_{0}, y_{0}\right)\right]>0$ for some $y_{0} \in G$. Fix any element $x_{0} \in E\left(q_{0}, y_{0}\right)$. Then $\lambda_{0}\left[E\left(q, q x_{0}\right)\right]=0$ for all $q \in N$ with $1 \leqq q<q_{0}$, so that there exists a compact subset $K_{0}$ of $E\left(q_{0}, y_{0}\right) \backslash\left\{\bigcup_{\bigcup^{\prime}=1}^{q_{0}-1} E\left(q, q x_{0}\right)\right\}$ with $\lambda_{0}\left(K_{0}\right)>0$. Replacing $K_{0}$ by the support of $\lambda_{0} \mid K_{0}$, we may assume that $K_{0}$ is perfect and satisfies (a). Evidently (b) holds. Suppose now that (c) holds for some $m \in N$ (note that (c) is trivial for $m=1$ ). Let $V_{1}, \cdots$, $V_{m}, V_{m+1}$ be $m+1$ disjoint, nonempty, open subsets of $K_{0}$. There are $m$ points $x_{1} \in V_{1}, \cdots, x_{m} \in V_{m}$ such that $x_{1}-x_{0}, \cdots, x_{m}-x_{0}$ are independent. Let $H$ be the subgroup of $G$ which is generated by $x_{0}, x_{1}$, $\cdots, x_{m}$. By minimality of $q_{0}$, we have $\lambda_{0}[E(q, y)]=0$ for all $q \in N$ 
with $1 \leqq q<q_{0}$ and all $y \in H$. Since $\lambda_{0}\left(V_{m+1}\right)>0$ by (a) and $H$ is at most countable, we can find an element $x_{m+1} \in V_{m+1}$ so that

$$
x_{m+1} \notin E(q, y) \quad\left(q=1,2, \cdots, q_{0}-1 ; y \in H\right) .
$$

It is now easy to prove that the elements $x_{1}-x_{0}, \cdots, x_{m}-x_{0}, x_{m+1}-x_{0}$ are independent. By induction on $m$, we obtain (c).

Suppose next that $\lambda_{0}[E(q, y)]=0$ for all $q \in N$ and all $y \in G$. Then $F=\left\{x \in \operatorname{supp} \lambda_{0}\right.$ :ord $\left.x<\infty\right\}$ has $\lambda_{0}$-measure zero, so that there exists a nonempty compact, perfect subset $K_{0}$ of $\left(\operatorname{supp} \lambda_{0}\right) \backslash F$ which satisfies (a). It is now easy to prove that (b) and (c) hold for $x_{0}=0$.

Lemma 3. Let $K$ be a totally disconnected, compact subset of $G$, and $\sigma$ a nonzero measure in $M_{c}^{+}(G)$ with supp $\sigma=K$. Then, for each compact subset $\hat{F}$ of $\hat{G}$ and $\varepsilon>0$, there exists a finite partition $\left\{K_{j}\right\}_{j=1}^{n}$ of $K$ into disjoint clopen subsets such that:
(i ) $0<\sigma\left(K_{j}\right)<\varepsilon$
$(j=1,2, \cdots, n)$;
(ii) $\left|\sum_{j=1}^{n} \sigma\left(K_{j}\right) \hat{\nu}_{j}(\chi)-\hat{\sigma}(\chi)\right|<\varepsilon$
$(\chi \in \hat{F})$

whenever $\nu_{j} \in M^{+}\left(K_{j}\right)$ and $\left\|\nu_{j}\right\|_{M}=1$ for all $j=1,2, \cdots, n$.

Proof. Since $\hat{F}$ is compact while $K$ is totally disconnected and compact, there is a finite partition $\left\{K_{j}\right\}_{j=1}^{n}$ of $K$ into disjoint clopen subsets which satisfies (i) and

$$
\sup \left\{|\chi(x)-\chi(y)|: x, y \in K_{\jmath}\right\}<\left(3\|\sigma\|_{M}\right)^{-1} \varepsilon
$$

for all $\chi \in \hat{F}$ and all $j=1,2, \cdots, n$. If $\nu_{j} \in M^{+}\left(K_{j}\right)$ and $\left\|\nu_{j}\right\|_{M}=1$ for $j=1,2, \cdots, n$, then we have

$$
\left|\sigma\left(K_{j}\right) \hat{\nu}_{j}(\chi)-\widehat{\sigma \mid K}_{j}(\chi)\right|<\|\sigma\|_{M}^{-1} \sigma\left(K_{j}\right) \varepsilon \quad(\chi \in \hat{F}) .
$$

To see this, take any $x_{j} \in K_{j}$. Then $\chi \in \hat{F}$ implies

$$
\begin{aligned}
& \left|\sigma\left(K_{j}\right) \widehat{\nu}_{j}(\chi)-{\widehat{\sigma \mid K_{j}}}_{j}(\chi)\right| \\
& \quad=\left|\sigma\left(K_{j}\right) \int_{K_{j}} \bar{\chi} d \nu_{j}-\int_{K_{j}} \bar{\chi} d \sigma\right| \\
& \quad \leqq \sigma\left(K_{j}\right) \int_{K_{j}}\left|\bar{\chi}-\bar{\chi}\left(x_{j}\right)\right| d \nu_{j}+\int_{K_{j}}\left|\bar{\chi}-\bar{\chi}\left(x_{j}\right)\right| d \sigma \\
& \quad \leqq 2 \sigma\left(K_{j}\right)\left(3\|\sigma\|_{M}\right)^{-1} \varepsilon<\|\sigma\|_{M}^{-1} \sigma\left(K_{j}\right) \varepsilon .
\end{aligned}
$$

Adding these inequalities for all $j$ 's, we obtain (ii).

To prove the following lemma, we need a definition. Let $K$ be a subset of $G$ whose elements have the same order $q_{0}\left(2 \leqq q_{0} \leqq \infty\right)$. Let also $L_{1}, L_{2}, \cdots, L_{n}$ be finitely many subsets of $K$, and $M$ any natural number. We say that $L_{1}, L_{2}, \cdots, L_{n}$ are $M$-independent if and only if $\sum_{j=1}^{n} m_{j} x_{j} \neq 0$ whenever $m_{j} \in Z$ (the integers), $\left|m_{j}\right|<q_{0}$, 
$x_{j} \in L_{j}(j=1,2, \cdots, n)$ and $0 \neq \sum_{j=1}^{k}\left|m_{j}\right|<M$.

Lemma 4. Suppose that $G$ is metrizable, and that $\left\{\lambda_{k}\right\}_{k=0}^{\infty}$ and $\left\{C_{k}\right\}_{k=0}^{\infty}$ are as in Theorem 1. Let also $x_{0} \in G$ and $K_{0} \subset \operatorname{supp} \lambda_{0}$ be as in Lemma 2. Then there exists a nonzero measure $\sigma \in M_{0}^{\perp}\left(K_{0}\right)$ such that $(\operatorname{supp} \sigma)-x_{0}$ is independent and

$$
\lambda_{k}\left[x+C_{k}+(\operatorname{supp} \sigma)_{1}\right]=0 \quad(x \in G ; k=0,1,2, \cdots) .
$$

Proof. Write

$$
\widehat{G}=\bigcup_{n=1}^{\infty} \hat{E}_{n} \quad \text { and } \quad C_{k}=\bigcup_{n=1}^{\infty} C_{k n} \quad(k=0,1,2, \cdots),
$$

where the $\hat{E}_{n}$ are compact subsets of $\hat{G}$ while the $C_{k n}$ are compact subsets of $G$ such that $C_{k n} \subset C_{k(n+1)}$ for all $k$ and $n$. (It is well-known that $G$ is metrizable if and only if $\hat{G}$ is $\sigma$-compact. See, for example, [4].) Let $\lambda$ be the measure in $M(G)$ defined by $\lambda(E)=\lambda_{0}\left[\left(E+x_{0}\right) \cap K_{0}\right]$ for all Borel subsets $E$ of $G$. Then, $0 \neq \lambda \in M_{0}^{+}(G)$ and the elements in $\operatorname{supp} \lambda=K_{0}-x_{0}$ have the same order $q_{0}$.

We shall now construct a sequence $\left(n_{p}\right)_{p=1}^{\infty}$ of natural numbers, a sequence $\left(\mathscr{F}_{n}\right)_{n=1}^{\infty}$ of finite collections of disjoint clopen subsets of $K_{0}-x_{0}$, a sequence $\left(\sigma_{n}\right)_{n=1}^{\infty}$ of probability measures in $L^{1}(\lambda)$, and a sequence $\left(\hat{F}_{n}\right)_{n=1}^{\infty}$ of compact subsets of $\hat{G}$. They will satisfy the following three conditions. Every $\sigma_{n}$ has the form

$$
\sigma_{n}=\sum_{I \in \mathscr{J}_{n}} a_{I} \lambda_{I}
$$

where each $a_{I}$ is a positive real number, $\lambda_{I}=\lambda \mid I$ the restriction of $\lambda$ to $I$, and

$$
\begin{gathered}
\left\|\sigma_{n}\right\|_{M}=\sum_{I \in \mathcal{J}_{n}} a_{I} \lambda(I)=1 . \\
\sup \left\{\widehat{\sigma_{n} \mid I}(\chi) \mid: \chi \in \widehat{G} \backslash \hat{F}_{n}\right\}<2^{-n} \sigma_{n}(I) \quad \forall I \in \mathscr{J}_{n} . \\
\hat{E}_{n} \subset \hat{F}_{n} .
\end{gathered}
$$

For $n=1$, such $\mathscr{F}_{1}, \sigma_{1}$, and $\hat{F}_{1}$ may be quite arbitrary. We set $n_{1}=1$, and suppose that $n_{p}, \mathscr{I}_{n_{p}}, \sigma_{n_{p}}$, and $\hat{F}_{n_{p}}$ have been constructed for some $p \in N$. Let $l_{p}=\operatorname{Card} \mathscr{F}_{n_{p}}$, and write

$$
\mathscr{J}_{n_{p}}=\left\{I_{i}\right\}_{i=1}^{l_{p}^{p}}=\left\{I_{i}^{p}\right\}_{i=1}^{l_{p}^{p}} \text {. }
$$

Let $M_{p}$ be the largest natural number such that

$$
\max \left\{\sigma_{n_{p}}(I): I \in \mathscr{J}_{n_{p}}\right\} \leqq M_{p}^{-2},
$$

and set 


$$
T_{p}=\left\{A \subset \mathcal{J}_{n_{p}}: 1 \leqq \operatorname{Card} A \leqq M_{p}\right\}=\left\{A_{r}\right\}_{r=1}^{s_{p}^{p}} .
$$

We may assume

$$
A_{r}=\left\{I_{r}\right\}=\left\{I_{r}^{p}\right\} \quad\left(r=1,2, \cdots, l_{p}\right) .
$$

We shall inductively construct the $\mathscr{F}_{n}, \sigma_{n}$, and $\hat{F}_{n}$ for all $n \in N$ with $n_{p}<n \leqq n_{p}+s_{p}$ as follows. Suppose that $\mathscr{F}_{n}, \sigma_{n}$ and $\hat{F}_{n}$ have been constructed for some $n=n_{p}+r-1\left(r=1,2, \cdots, s_{p}\right)$, and put

$$
\mathscr{K}_{n}=\left\{I \in \mathscr{F}_{n}: I \subset J \text { for some } J \in A_{r}\right\} \text {. }
$$

We can find (finite) collections $\left\{b_{\jmath}^{K}\right\}_{j}$ of real numbers and collections $\left\{L_{j}^{K}\right\}_{j}$ of disjoint clopen subsets of $K \in \mathscr{K}_{n}$ which satisfy the following six conditions:

$$
\begin{array}{ll}
0<b_{j}^{K} \sigma_{n}\left(L_{j}^{K}\right)<2^{-1} \sigma_{n}(K) & \forall K \in \mathscr{K}_{n} \text { and } \forall_{j} ; \\
\sum_{j} b_{j}^{K} \sigma_{n}\left(L_{j}^{K}\right)=\sigma_{n}(K) & \forall K \in \mathscr{K}_{n} ;
\end{array}
$$

$$
\left|\sum_{j} b_{j}^{K} \widehat{\sigma_{n} \mid L_{j}^{K}}(\chi)-\widehat{\sigma_{n} \mid K}(\chi)\right|<2^{-n} \sigma_{n}(K) \quad \forall K \in \mathscr{K}_{n} \text { and } \forall \chi \in \hat{F}_{n} \text {; }
$$

$$
\sum_{j} \operatorname{dia}\left(L_{j}^{K}\right)<n^{-1} \quad \forall K \in \mathscr{\varkappa}_{n}^{\prime} ;
$$

The sets $\left\{L_{j}^{K}\right\}_{K, j}$ are $M_{p}$-independent;

$$
\begin{aligned}
\sup _{x \in G} \lambda_{k}\left[x+C_{k n}+\left(\bigcup_{\jmath} L_{j}^{K}\right)_{1}\right] & <\left(n l_{p}\right)^{-1} \\
& \forall K \in \mathscr{K}_{n} \text { and } \forall k=0,1, \cdots, n .
\end{aligned}
$$

The above conditions are met as follows: For each $K \in \mathscr{K}_{n}$, apply Lemma 3 to $\sigma=\sigma_{n} \mid K, \varepsilon=2^{-n} \sigma_{n}(K)$ and $\hat{F}=\hat{F}_{n}$. Let $\left\{K_{j}\right\}_{j}$ be a finite partition of $K$ as in Lemma 3. Using property (c) in Lemma 2, we can find $x_{j}^{K} \in K_{j}$ so that $\bigcup\left\{\left\{x_{j}^{K}\right\}_{0}: K \in \mathscr{Y}_{n}\right\}$ is independent. If we choose $L_{j}^{K} \subset K_{j}$ so that $x_{3}^{K} \in L_{3}^{K}$ and the diameter of each $L_{j}^{K}$ is "sufficiently small", then (8) and (9) hold and so does (10) by Lemma 1. Finally, it suffices to set $b_{j}^{K}=\sigma_{n}\left(K_{j}\right) / \sigma_{n}\left(L_{j}^{K}\right)$.

We now define

$$
\begin{gathered}
\mathscr{L}_{n}=\mathscr{F}_{n} \mid \mathscr{K}_{n}, \mathscr{J}_{n+1}=\mathscr{L}_{n} \cup\left(\bigcup_{K \in \mathscr{K}_{n}}\left\{L_{\jmath}^{K}\right\}_{j}\right) ; \\
\theta_{n+1}=\sum_{I \in \mathscr{E}_{n}} a_{I} \lambda_{I}+\sum_{K \in \mathscr{N}_{n}} \sum_{j} b_{\jmath}^{K} \sigma_{n} \mid L_{\jmath}^{K},
\end{gathered}
$$

and take a compact subset $\hat{F}_{n+1}$ of $\hat{G}$, with $\hat{F}_{n+1} \supset \hat{E}_{n+1} \cup \hat{F}_{n}$, so that (ii) holds with $n$ replaced by $n+1$.

We repeat the above process with $n_{p}$ replaced by $n_{p+1}=n_{p}+s_{p}$, which completes our induction. Let $\sigma_{\infty}$ be a weak-* cluster point of 
$\left(\sigma_{n}\right)_{n=1}^{\infty}$ in $M(G)$, and $\sigma$ the measure in $M(G)$ defined by $\sigma(E)=$ $\sigma_{\infty}\left(E-x_{0}\right)$ for all Borel sets $E$ in $G$. We claim that $\sigma$ has the required properties.

First note that

$$
\bigcup_{I \in \cup_{n+1}} I \subset \bigcup_{I \in \cup_{n}} I=\operatorname{supp} \sigma_{n} \subset K_{0}-x_{0},
$$

and so we have

$$
\sigma_{\infty} \geqq 0, \quad \sigma_{\infty}(G)=1 \quad \text { and } \quad \operatorname{supp} \sigma_{\infty} \subset \bigcap_{n=1}^{\infty}\left(\bigcup_{I \in \sim_{n}} I\right) .
$$

Let $p \in N$ be given. It is easily seen from (3), (4), and (11) that

$$
\mathscr{J}_{n_{p}+l_{p}}=\left\{L_{j}^{Y_{1}}\right\}_{j} \cup\left\{L_{j}^{T_{2}}\right\}_{j} \cup \cdots \cup\left\{L_{j}^{I}\right\}_{j} \text {, where } l=l_{p} \text {. }
$$

This, combined with (10) and (13), shows

$$
\begin{aligned}
\lambda_{k}[x+ & \left.C_{k n_{p}}+\left(\operatorname{supp} \sigma_{\infty}\right)_{1}\right] \\
& \leqq \sum_{i=1}^{l_{p}} \lambda_{k}\left[x+C_{k n_{p}}+\left(\bigcup_{j} L_{j}^{I_{i}}\right)_{1}\right] \leqq l_{p} \cdot\left(n_{p} l_{p}\right)^{-1}=n_{p}^{-1}
\end{aligned}
$$

for all $x \in G$ and all $k=0,1, \cdots, n_{p}$. (Note that $C_{k n} \subset C_{k(n+1)}$ for all $k$ and $n$.) Thus, fixing $x \in G$ and $k \in\{0,1,2, \cdots\}$, and letting $p \rightarrow \infty$, we have

$$
\lambda_{k}\left[x+C_{k}+\left(\operatorname{supp} \sigma_{\infty}\right)_{1}\right]=0 \quad(x \in G ; k=0,1,2, \cdots) .
$$

But evidently $\operatorname{supp} \sigma=\left(\operatorname{supp} \sigma_{\infty}\right)+x_{0}$, and so $\left(\mathrm{P}_{1}\right)$ holds.

It remains to show that $\hat{\sigma}$ vanishes at infinity and that $(\operatorname{supp} \sigma)-x_{0}$ is independent. Although these are proved in [5: Ch. XIII, 151-153 and 155-156], we give their proofs to make the paper self-contained.

Suppose $n_{p} \leqq n<n_{p+1}(p, n \in N)$, and write $n=n_{p}+r-1(r=$ $\left.1,2, \cdots, s_{p}\right)$. Then we have

$$
\sum_{K \in \mathscr{W}_{n}} \sigma_{n}(K)=\sum_{J \in A_{r}} \sigma_{n_{p}}(J) \leqq\left(\operatorname{Card} A_{r}\right) \cdot \max _{J \in A_{r}} \sigma_{n_{p}}(J) \leqq M_{p}^{-1} .
$$

Here the equality follows from (4), (6) and (12) while the last inequality follows from (1) and (2). If $\chi \in \hat{F}_{n}$, then

$$
\begin{aligned}
\left|\hat{\sigma}_{n+1}(\chi)-\hat{\sigma}_{n}(\chi)\right| & \leqq \sum_{K \in \mathscr{N}_{n}}\left|\sum_{j} b_{j}^{K} \widehat{\sigma_{n} \mid L_{j}^{K}}(\gamma)-\widehat{\sigma_{n} \mid K}(\chi)\right| \\
& <\sum_{K \in \mathscr{K}_{n}} 2^{-n} \sigma_{n}(K) \leqq 2^{-n}
\end{aligned}
$$

by (i), (12) and (7). It follows that

$$
\left|\hat{\sigma}_{\infty}(\chi)-\hat{\sigma}_{n+1}(\chi)\right|<2^{-n} \quad \forall \chi \in \hat{F}_{n+1},
$$

since $\hat{F}_{n} \subset \hat{F}_{n+1} \subset \cdots$ by construction. For $\chi \in \hat{G} \backslash \hat{F}_{n}$, we have 


$$
\begin{aligned}
\left|\hat{\sigma}_{n+1}(\chi)\right| & \leqq \sum_{I \in \mathscr{L}_{n}}\left|a_{I} \hat{\lambda}_{I}(\chi)\right|+\sum_{K \in \mathscr{T}_{n}} \sum_{j} b_{j}^{K} \sigma_{n}\left(L_{j}^{K}\right) \\
& \leqq \sum_{I \in \cup_{n}}\left|\widehat{\sigma_{n} \mid I}(\chi)\right|+\sum_{K \in \mathscr{I}_{n}} \sigma_{n}(K) \\
& \leqq 2^{-n} \sum_{I \in \mho_{n}} \sigma_{n}(I)+M_{p}^{-1}=2^{-n}+M_{p}^{-1}
\end{aligned}
$$

by (12), (i), (6), (ii) and (14). Hence

$$
\left|\hat{\sigma}_{\infty}(\chi)\right| \leqq 2^{-n}+2^{-n}+M_{p}^{-1} \quad \forall \chi \in \hat{F}_{n+1} \mid \hat{F}_{n} .
$$

But $\hat{G}=\bigcup_{n=1}^{\infty} \hat{F}_{n}$ by (iii) and $\lim _{p} M_{p}=\infty$ by construction. Thus the above inequality shows that $\hat{\sigma}_{\infty} \in C_{0}(\hat{G})$, or equivalently, that $\hat{\sigma} \in C_{0}(\hat{G})$.

Finally we prove that $(\operatorname{supp} \sigma)-x_{0}=\operatorname{supp} \sigma_{\infty}$ is independent. Let $x_{1}, x_{2}, \cdots, x_{t}$ be distinct elements of $\operatorname{supp} \sigma_{\infty}$. It is easy to see that

$$
\max _{I \in \mathscr{I}_{n}} \operatorname{dia}(I) \longrightarrow 0 \text { as } n \longrightarrow \infty \text {. }
$$

Therefore, there is an $n_{0} \in N$ such that $x_{1}, x_{2}, \cdots, x_{t}$ belong to distinct sets in $\mathscr{I}_{n}$ whenever $n \geqq n_{0}$. Take any $p \in N$ so that $n_{p} \geqq n_{0}$ and $M_{p}>t$, and let

$$
A=\left\{I \in \mathscr{F}_{n_{p}}: I \text { contains some } x_{n}\right\} .
$$

Then $1 \leqq \operatorname{Card} A=t<M_{p}$; hence $A=A_{r}$ for some $r=1,2, \cdots, s_{p}$. Thus $x_{1}, x_{2}, \cdots, x_{t}$ belong to distinct sets in $\bigcup\left\{\left\{L_{j}^{K}\right\}_{j}: K \in \mathscr{K}_{n}\right\}$, where $n=n_{p}+r-1$. It follows from (9) that $x_{1}, x_{2}, \cdots, x_{t}$ are $M_{p}$-independent. Since $p$ can be taken as large as one pleases, we conclude that $x_{1}, x_{2}, \cdots, x_{t}$ are independent.

This establishes Lemma 4.

Lemma 5. Let $G,\left\{\lambda_{k}\right\}_{k=0}^{\infty}$ and $\left\{C_{k}\right\}_{k=0}^{\infty}$ be as in Lemma 4. Let also $\left\{K_{j}\right\}_{j=1}^{m}$ be finitely many, disjoint, compact subsets of $G$ such that $M_{0}\left(K_{j}\right) \neq 0$ for all $j=1,2, \cdots, m$. Then, for each $n \in N$, there exist $m$ nonzero measures $\mu_{j} \in M_{0}^{+}\left(K_{j}\right)$ such that

$$
\lambda_{k}\left[x+C_{k}+\left(\bigcup_{j=1}^{m} \operatorname{supp} \mu_{j}\right)_{n}\right]=0 \quad(x \in G ; k=0,1,2, \cdots) .
$$

Proof. For each $j=1,2, \cdots, m$, choose and fix a measure $\tau_{j} \in$ $M_{0}^{+}\left(K_{j}\right)$ with $\left\|\tau_{j}\right\|_{M}=m^{-1}$ whose support is totally disconnected. In the proof of Lemma 4 , replace $\lambda$ and $K_{0}-x_{0}$ by $\lambda^{\prime}=\sum_{j=1}^{m} \tau_{j}$ and $\bigcup_{j=1}^{m} \operatorname{supp} \tau_{j}$, respectively; take off condition (9); and let $\sigma_{\infty}$ be any measure constructed as there with $\sigma_{1}=\lambda^{\prime}$ and $\mathscr{J}_{1}=\left\{\operatorname{supp} \tau_{j}\right\}_{j=1}^{m}$. Then

$$
0 \neq \mu_{j}=\sigma_{\infty} \mid K_{j} \in M_{0}^{+}\left(K_{j}\right) \quad(j=1,2, \cdots, m),
$$

and $\left\{\mu_{j}\right\}_{j=1}^{m}$ satisfy $\left(\mathrm{P}_{1}\right)$. We repeat the same argument replacing 
$\left\{C_{k}\right\}_{k=0}^{\infty}$ and $\left\{K_{j}\right\}_{j=1}^{m}$ by $\left\{C_{k}+\left(\bigcup_{j=1}^{m} \operatorname{supp} \mu_{j}\right)_{1}\right\}_{k=0}^{\infty}$ and $\left\{\operatorname{supp} \mu_{j}\right\}_{j=1}^{m}$, respectively; and continue this process. At the $n$th step, we will obtain $m$ nonzero measures satisfying the required condition. This completes the proof.

Proof of Theorem 1 for metrizable groups. Suppose that $G$ is a given metrizable group. Let $\left\{\lambda_{k}\right\}_{k=0}^{\infty},\left\{C_{k}\right\}_{k=0}^{\infty}, x_{0}$, and $K_{0}$ be as in Lemma 4. Let also $\widehat{G}=\bigcup_{k=1}^{\infty} \hat{E}_{n}$ be as in the proof of Lemma 4 . We construct a sequence $\left(\mathscr{F}_{n}\right)_{n=1}^{\infty}$ of finite collections of disjoint compact subsets of $K_{0}$, a sequence $\left(\sigma_{n}\right)_{n=1}^{\infty}$ of probability measures in $M_{0}\left(K_{0}\right)$, and a consequence $\left(\hat{F}_{n}\right)_{n=1}^{\infty}$ of compact subsets of $\widehat{G}$. They satisfy the following four conditions. Every $\sigma_{n}$ has the form

$$
\sigma_{n}=\sum_{I \in \cup_{n}} \alpha_{I} \mu_{I},
$$

where each $\alpha_{I}$ is a real positive number, $\mu_{I}$ a probability measure in $M_{0}(I)$ with $\operatorname{supp} \mu_{I}=I$, and

$$
\left\|\sigma_{n}\right\|_{M}=\sum_{I \in \bigcup_{n}} \alpha_{I}=1 \text {. }
$$

$$
\begin{aligned}
& \sup \left\{\left|\hat{\mu}_{I}(\chi)\right|: \chi \in \hat{G} \backslash \hat{F}_{n}\right\}<2^{-n} a_{I} \quad \forall I \in \mathscr{F}_{n} . \\
& \hat{E}_{n} \subset \hat{F}_{n} . \\
& \begin{aligned}
\lambda_{k}\left[x+C_{k}+\left(K_{1}\right)_{1}+\left(K_{2}\right)_{2}+\cdots+\right. & \left.\left(K_{n}\right)_{n}\right]=0 \\
& (x \in G ; k=0,1,2, \cdots),
\end{aligned}
\end{aligned}
$$

where $K_{1}=\bigcup\left\{I: I \in \mathscr{F}_{1}\right\}$ and $K_{n}=\bigcup\left\{I \in \mathscr{F}_{n}: I \notin \mathscr{F}_{n-1}\right\}$ for $n=2,3, \cdots$.

For $n=1$, we apply Lemma 4 to obtain a probability measure $\sigma_{1} \in M_{0}\left(K_{0}\right)$ such that $\left(\operatorname{supp} \sigma_{1}\right)-x_{0}$ is independent and

$$
\lambda_{k}\left[x+C_{k}+\left(\operatorname{supp} \sigma_{1}\right)_{1}\right]=0 \quad(x \in G ; k=0,1,2, \cdots) .
$$

Set $\mathscr{J}_{1}=\left\{I=\operatorname{supp} \sigma_{1}\right\}, \mu_{I}=\sigma_{1}, a_{I}=1$, and take any compact subset $\hat{F}_{1}$ of $\hat{G}$ satisfying (ii) and (iii) for $n=1$.

Suppose that $\left(\mathscr{F}_{2}\right)_{i=1}^{n},\left(\sigma_{2}\right)_{i=1}^{n}$ and $\left(\hat{F}_{i}\right)_{i=1}^{n}$ have been constructed for some $n \in N$. Choose and fix any $I_{n} \in \mathscr{F}_{n}$ with

$$
a_{I_{n}}=\sup \left\{a_{I}: I \in \mathscr{J}_{n}\right\} \text {. }
$$

Applying Lemmas 3 and 5, we can find a (finite) collection $\left\{b_{n j}\right\}_{j}$ of real numbers, a collection $\left\{L_{n j}\right\}_{j}$ of disjoint compact subsets of $I_{n}$, and a collection $\left\{\mu_{n j}\right\}_{j}$ of probability measures in $M_{0}\left(I_{n}\right)$ with supp $\mu_{n j}=$ $L_{n j}$ which satisfy the following four conditions.

$$
0<b_{n j}<n^{-1} \cdot \min _{I \in \mathcal{V}_{n}} a_{I} \quad \forall j \text {. }
$$




$$
\begin{gathered}
\sum_{j} b_{n j}=a_{I_{n}} \\
\left|\sum_{j} b_{n j} \hat{\mu}_{n j}(\chi)-a_{I_{n}} \hat{\mu}_{I_{n}}(\chi)\right|<2^{-n} \quad \forall \chi \in \hat{F}_{n} . \\
\lambda_{k}\left[x+C_{k}+\left(K_{1}\right)_{1}+\cdots+\left(K_{n}\right)_{n}+\left(\bigcup_{j} L_{n j}\right)_{n+1}\right]=0 \quad \forall x \in G
\end{gathered}
$$

for all $k=0,1,2, \cdots$. Put $\mathscr{J}_{n+1}=\left(\mathscr{F}_{n} \backslash\left\{I_{n}\right\}\right) \cup\left\{L_{n j}\right\}_{j}$, and $a_{I}=b_{n j}$, $\mu_{I}=\mu_{n j}$ for $I=L_{n j} \forall j$. Define $\sigma_{n+1}$ by the right-hand side of (i) with $n$ replaced by $n+1$. Finally, we take any compact subset $\hat{F}_{n+1}$ of $\hat{G}$, with $\widehat{F}_{n+1} \supset \widehat{E}_{n+1} \cup \widehat{F}_{n}$, so that (ii) holds with $n$ replaced by $n+1$.

This completes the induction. Let $\sigma$ be a weak-* cluster point of $\left(\sigma_{n}\right)_{n=1}^{\infty}$ in $M(G)$. Then it is easy to prove that $\sigma$ has all the required properties (see [5: Ch. XIII, 151-153]). This establishes Theorem 1 for metrizable groups.

To prove the general case, we need one more lemma.

Lemma 6. Let $\left\{\lambda_{k}\right\}_{k=0}^{\infty}$ and $\left\{C_{k}\right\}_{k=0}^{\infty}$ be as in Theorem 1. Then, given a $\sigma$-compact subset $\hat{F}$ of $\hat{G}$, we can find a $\sigma$-compact, noncompact, open subgroup $\Gamma$ of $\hat{G}$ so that $\hat{F} \subset \Gamma$ and

$$
\lambda_{k}\left[x+C_{k}+H_{\Gamma}\right]=0 \quad(x \in G ; k=0,1,2, \cdots),
$$

where $H_{\Gamma}$ denotes the annihilator of $\Gamma$.

Proof. Let $C_{k n}$ be as in the proof of Lemma 4, and let $\mathscr{F}$ be the family of all $\sigma$-compact, noncompact, open subgroups of $\hat{G}$ which contain $\hat{F}$. Since every $C_{k n}$ is compact, we have

$$
C_{k n}=\bigcap\left\{C_{k n}+H_{\Gamma}: \Gamma \in \mathscr{F}\right\} \quad(k=0,1,2, \cdots ; n=1,2, \cdots) .
$$

Applying Lemma 1, we can find neighborhoods $V_{k n}$ of 0 so that

$$
\lambda_{k}\left[x+C_{k n}+V_{k n}\right]<n^{-1} \quad(k=0,1,2, \cdots ; n=1,2, \cdots: x \in G) .
$$

By (1), there exist subgroups $\Gamma_{k n}$ in $\mathscr{F}$ such that

$$
C_{k n}+H_{k n} \subset C_{k n}+V_{k n} \quad(k=0,1,2, \cdots ; n=1,2, \cdots),
$$

where $H_{k n}$ is the annihilator of $\Gamma_{k n}$. Let $\Gamma$ be any subgroup in $\mathscr{F}$ which contains all $\Gamma_{k n}$. Then, it follows from (2) and (3) that (i) holds. This completes the proof.

Proof of Theorem 1 for general groups. Let $G$ be an arbitrary nondiscrete LCA group, and let $\left\{\lambda_{k}\right\}_{k=0}^{\infty}$ and $\left\{C_{k}\right\}_{k=0}^{\infty}$ be as in Theorem 1. For $\hat{F}=\left\{\chi \in \hat{G}: \hat{\lambda}_{0}(\chi) \neq 0\right\}$, take a $\Gamma \subset G$ as in Lemma 6. Setting 
$H=H_{\Gamma}$, we denote by $\pi$ and $m_{H}$ the natural mapping of $G$ onto $G_{0}=G / H$ and the Haar measure of $H$ with $m_{H}(H)=1$, respectively. For each $\mu \in M(G)$, define a measure $\mu^{\prime} \in M\left(G_{0}\right)$ by setting

$$
\int_{G_{0}} f d \mu^{\prime}=\int_{G} f \circ \pi d \mu \quad \forall f \in C_{0}\left(G_{0}\right) .
$$

Identifying $\Gamma$ with $\hat{G}_{0}$ in the usual way, we see $\hat{\mu}^{\prime}=\hat{\mu} \mid \Gamma$ for all $\mu \in M(G)$, so that $0 \neq \lambda_{0}^{\prime} \in M_{0}\left(G_{0}\right)$. On the other hand, we have

$$
\lambda_{k}^{\prime}\left[x^{\prime}+C_{k}^{\prime}\right]=0 \quad\left(x^{\prime} \in G_{0} ; k=0,1,2, \cdots\right)
$$

by (i) in Lemma 6 and (1), where $C_{k}^{\prime}=\pi\left(C_{k}\right)$. Therefore $\left\{\lambda_{k}^{\prime}\right\}_{k=0}^{\infty} \subset M_{c}^{+}(G)$, and we can apply our result for metrizable groups to find a nonzero measure $\sigma^{\prime} \in M_{0}^{+}\left(\operatorname{supp} \lambda_{0}^{\prime}\right)$ with compact support such that

$$
\lambda_{k}^{\prime}\left[x^{\prime}+C_{k}^{\prime}+G_{p}\left(\operatorname{supp} \sigma^{\prime}\right)\right]=0 \quad\left(x^{\prime} \in G_{0} ; k=0,1,2, \cdots\right) .
$$

Now define a measure $\sigma \in M(G)$ by setting

$$
\int_{G} f d \sigma=\int_{G_{0}}\left\{\int_{I I} f(x+t) d m_{I I}(t)\right\} d \sigma^{\prime}\left(x^{\prime}\right) \quad \forall f \in C_{0}(G) .
$$

As is easily seen, we then have

$$
\operatorname{supp} \sigma=\pi^{-1}\left[\operatorname{supp} \sigma^{\prime}\right] \text { and } \operatorname{supp} \lambda_{0}=\pi^{-1}\left[\operatorname{supp} \lambda_{0}^{\prime}\right]
$$

(note that $\sigma * m_{I I}=\sigma$ and $\lambda_{0} * m_{I I}=\lambda_{0}$ ). It is also easy to check that $0 \neq \sigma \in M_{0}^{+}(G)$, that $\operatorname{supp} \sigma$ is a compact subset of supp $\lambda_{0}$, and that

$$
\lambda_{k}\left[x+C_{k}+G_{p}(\operatorname{supp} \sigma)\right]=\lambda_{k}^{\prime}\left[x^{\prime}+C_{k}^{\prime}+G_{p}\left(\operatorname{supp} \sigma^{\prime}\right)\right]=0
$$

for all $x \in G$ and all $k=0,1,2, \cdots$.

This establishes Theorem 1.

Proof of Corollary. Let $Y$ be as in the present Corollary. Setting $C_{k}=\{0\}$ for all $k$ and applying Theorem 1 to $\left\{\left|\lambda_{k}\right|\right\}_{k=0}^{\infty}$, we obtain a nonzero measure $\sigma \in M_{0}^{+}\left(\operatorname{supp} \lambda_{0}\right)$ with compact support such that

$$
|\tau|\left[x+G_{p}(\operatorname{supp} \sigma)\right]=0 \quad(x \in G)
$$

holds for all $\tau \in \bigcup_{k=0}^{\infty} L^{1}\left(\lambda_{k}\right)$. But then we have

$$
\begin{gathered}
|\nu * \tau|\left[x+G_{p}(\operatorname{supp} \sigma)\right] \leqq(|\nu| *|\tau|)\left[x+G_{p}(\operatorname{supp} \sigma)\right] \\
\leqq \int_{G}|\tau|\left[x-y+G_{p}(\operatorname{supp} \sigma)\right] d|\nu|(y)=0
\end{gathered}
$$

for all $x \in G$ whenever $\nu \in M(G)$ and $\tau \in \bigcup_{k=0}^{\infty} L^{1}\left(\lambda_{k}\right)$. Since the ideal $Y$ is generated by $\bigcup_{k=0}^{\infty} L^{1}\left(\lambda_{k}\right)$, this implies that (1) holds for all $\tau \in Y$.

The last statement in the Corollary is now trivial and the proof is complete. 
To prove Theorem 2, we need some notation. Let $\mathscr{T}$ be a nonempty family of (locally) Borel measurable subgroups of $G$ such that for any countable subfamily $\mathscr{T}_{0}$ of $\mathscr{T}$ there exists a subgroup $H \in \mathscr{T}$ which contains all $L \in \mathscr{T}_{0}$. Define

$$
I(\mathscr{T})=\{\mu \in M(G):|\mu|(x+H)=0 \forall x \in G \text { and } \forall H \in \mathscr{T}\}
$$

and

$$
\begin{aligned}
\mathscr{R}(\mathscr{T})= & \{\nu \in M(G):|\nu|(G \backslash(D+H))=0 \text { for some countable } \\
& D \subset G \text { and some } H \in \mathscr{T}\} .
\end{aligned}
$$

Then it is easy to prove the following (cf. [1]):

(a) $I(\mathscr{T})$ is a closed ideal in $M(G)$ such that $I(\mathscr{T})^{*}=I(\mathscr{T})$.

(b) $\mathscr{R}(\mathscr{T})$ is a closed subalgebra of $M(G)$ such that $\mathscr{R}(\mathscr{T})^{*}=$ $\mathscr{R}(\mathscr{T})$.

(c) $M(G)=I(\mathscr{T})+\mathscr{R}(\mathscr{T})$ and $I(\mathscr{T}) \cap \mathscr{R}(\mathscr{T})=\{0\}$.

We denote by $\Phi_{,}$the projection of $M(G)$ onto $\mathscr{R}(\mathscr{T})$ which is induced by the direct sum decomposition $M(G)=I(\mathscr{T})+\mathscr{R}(\mathscr{T})$. Note that $\Phi_{\mathscr{T}}$ is a ${ }^{*}$-homomorphism of $M(G)$ onto $\mathscr{R}(\mathscr{T})$.

Proof of Theorem 2. Let $\left\{\lambda_{k}\right\}_{k=0}^{\infty}$ and $Y$ be as in Corollary; without loss of generality, we may assume that $\lambda_{k} \geqq 0$ for all $k$. By Lemma 6 , there is a $\sigma$-compact, noncompact, open subgroup $\Gamma$ of $\widehat{G}$ such that

$$
\lambda_{k}(x+H)=0 \quad(x \in G ; k=0,1,2, \cdots),
$$

where $H$ is the annihilator of $\Gamma$. Let $G_{0}=G / H$, and let $\mu \rightarrow \mu^{\prime}$ be the mapping of $M(G)$ onto $M\left(G_{0}\right)$ defined in the proof of Theorem 1 for general groups. Note that $\mu \rightarrow \mu^{\prime}$ is a *homomorphism. Since (1) implies $\lambda_{k}^{\prime} \in M_{c}(G)$ for all $k$, Theorem 1 assures that there exists a nonzero measure $\sigma^{\prime} \in M_{0}^{+}\left(G_{0}\right)$ with compact support such that $K^{\prime}=$ $\operatorname{supp} \sigma^{\prime}$ is independent and

$$
\lambda_{k}^{\prime}\left[x^{\prime}+G_{p}\left(K^{\prime}\right)\right]=0 \quad\left(x^{\prime} \in G_{0} ; k=0,1,2, \cdots\right) .
$$

Let $\omega_{1}$ be the first countable ordinal and let $W=\{1,2, \cdots\}$ be the well-ordered set consisting of all ordinals smaller than $\omega_{1}$. We now construct a family $\left\{L_{\alpha}^{\prime}: \alpha \in W\right\}$ of disjoint compact subsets of $K^{\prime}$ such that

$$
M_{0}\left(L_{\alpha}^{\prime}\right) \neq\{0\} \quad \text { and } \quad \sigma^{\prime}\left(L_{\alpha}^{\prime}\right)=0
$$

for all $\alpha \in W$. First, by Theorem 1, there exists a compact subset $L_{1}^{\prime}$ of $K^{\prime}$ having property (3). Let $\beta \in W, \beta \geqq 2$, and suppose that $L_{\alpha}^{\prime}$ has been constructed for all $\alpha \in W$ with $\alpha<\beta$. Then $E_{\beta}^{\prime}=$ $\bigcup\left\{L_{\alpha}^{\prime}: \alpha<\beta\right\}$ is $\sigma$-compact, and by (3), has $\sigma^{\prime}$-measure zero. Therefore, there exists a compact subset $F_{\beta}^{\prime}$ of $K^{\prime} \backslash E_{\beta}^{\prime}$ having positive $\sigma^{\prime}$ - 
measure. Applying Theorem 1 again, we can find a compact subset $L_{\beta}^{\prime}$ of $F_{\beta}^{\prime}$ so that (3) holds for $\alpha=\beta$. By transfinite induction, we obtain a family $\left\{L_{\alpha}^{\prime}: \alpha \in W\right\}$ of disjoint compact subsets of $K^{\prime}$ satisfying (3).

Let $\mathscr{P}(W)$ be the family of all nonempty subsets of $W$; hence Card $\mathscr{P}(W)=2^{\omega}$, where $\omega$ denotes the smallest uncountable cardinal. For each $A \in \mathscr{P}(W)$ and $\chi \in \Gamma$, we construct a complex homomorphism $\Psi_{A x}$ of $M(G)$ as follows. Let $\mathscr{T}=\mathscr{T}_{A}$ be the family of subgroups of $G$ each of which is generated by $\bigcup\left\{L_{\alpha}^{\prime}: \alpha \in B\right\}$ for some countable subset $B$ of $A$. We define $\Psi_{A x}$ by setting

$$
\Psi_{A \chi}(\mu)=\widehat{\Phi_{\sigma}\left((\chi \mu)^{\prime}\right)}(1) \quad(\mu \in M(G)) .
$$

It is easy to see that $\Psi_{A \chi}$ is a symmetric complex homomorphism of $M(G)$. Also $\Psi_{A \chi} \neq 0$ because

$$
\Psi_{A \chi}\left(\delta_{x}\right)=\chi(x) \neq 0 \quad(x \in G),
$$

where $\delta_{x}$ denotes the unit mass at $x$.

Fixing an $A \in \mathscr{P}(W)$ and $\chi \in \Gamma$, we now prove

$$
\bigcup\left\{L^{1}(\mu): \mu \in Y\right\} \cup M_{a}(G) \subset \operatorname{Ker} \Psi_{A \chi} \quad \text { but } M_{0}(G) \not \subset \operatorname{Ker} \Psi_{A \chi} .
$$

First note that $\nu \in M^{+}(G)$ and $\mu \in L^{1}(\nu)$ imply $\mu^{\prime} \in L^{1}\left(\nu^{\prime}\right)$. In fact, if $w$ is a bounded Borel function on $G$, we have

$$
\begin{aligned}
\left|\int_{G_{0}} f d(w \nu)^{\prime}\right| & =\left|\int_{G}(f \circ \pi) w d \nu\right| \leqq \int_{G}(|f| \circ \pi)|w| d \nu \\
& \leqq\|w\|_{\infty} \int_{G}(|f| \circ \pi) d \nu=\|w\|_{\infty} \cdot\|f\|_{L^{1}\left(\nu^{\prime}\right)}
\end{aligned}
$$

for all $f \in C_{0}\left(G_{0}\right)$, so that $(w \nu)^{\prime} \in L^{1}\left(\nu^{\prime}\right)$. Since the mapping $\tau \rightarrow \tau^{\prime}$ is norm-decreasing, we see

$$
\inf \left\{\left\|\mu^{\prime}-\tau^{\prime}\right\|_{M}: \tau^{\prime} \in L^{1}\left(\nu^{\prime}\right)\right\} \leqq\left\|\mu^{\prime}-(w \nu)^{\prime}\right\|_{M} \leqq\|\mu-w \nu\|_{M} .
$$

Since $w$ was arbitrary and $\mu \in L^{1}(\nu)$, this implies $\mu^{\prime} \in L^{1}\left(\nu^{\prime}\right)$. Suppose now that $\nu \in M(G)$ and $\lambda \in L^{1}\left(\lambda_{k}\right)$ for some $k$. Then, the above observation and (2) show

$$
\begin{aligned}
\left|(\nu * \lambda)^{\prime}\right|\left(x^{\prime}+T^{\prime}\right) & =\left|\nu^{\prime} * \lambda^{\prime}\right|\left(x^{\prime}+T^{\prime}\right) \leqq\left(\left|\nu^{\prime}\right| *\left|\lambda^{\prime}\right|\right)\left(x^{\prime}+T^{\prime}\right) \\
& \leqq\left(\left|\nu^{\prime}\right| *\left|\lambda^{\prime}\right|\right)\left[x^{\prime}+G_{p}\left(K^{\prime}\right)\right]=0
\end{aligned}
$$

for all $x^{\prime} \in G_{0}$ and all $T^{\prime} \in \mathscr{T}=\mathscr{T}_{A}$. Since the linear span of the sets $M(G) * L^{1}\left(\lambda_{k}\right), k=0,1,2, \cdots$, is dense in $Y$, it follows that

$$
\left|\tau^{\prime}\right|\left(x^{\prime}+T^{\prime}\right)=0 \quad\left(x^{\prime} \in G_{0} ; T^{\prime} \in \mathscr{T}\right)
$$

holds for all $\tau \in Y$, and so for all $\tau \in \bigcup\left\{L^{1}(\mu): \mu \in Y\right\}$. Therefore we have 


\section{$\bigcup\left\{L^{1}(\mu): \mu \in Y\right\} \subset \operatorname{Ker} \Psi_{A \chi} \cdot$}

Note now that $\lambda_{0}^{\prime} \neq 0$, and so $G_{p}\left(\operatorname{supp} \sigma^{\prime}\right)$ has no interior point by (2); hence the Haar measure of $G_{p}\left(\operatorname{supp} \sigma^{\prime}\right)$ is zero. Since $M_{a}(G)^{\prime}=M_{a}\left(G_{0}\right)$, it follows that $M_{a}(G) \subset \operatorname{Ker} \Psi_{A \chi}$. To prove that $M_{0}(G) \not \subset \operatorname{Ker} \Psi_{A \chi}$, take any $\alpha \in A$. Then $L_{\alpha}^{\prime} \subset G_{p}\left(L_{\alpha}^{\prime}\right) \in \mathscr{T}$, and so $\Phi\left[M_{0}\left(L_{\alpha}^{\prime}\right)\right]=M_{0}\left(L_{\alpha}^{\prime}\right) \neq\{0\}$. This establishes (6) because $M_{0}(G)^{\prime}=M_{0}\left(G_{0}\right)$.

Finally, take any $A, B \in \mathscr{P}(W)$ and any $\chi, \gamma \in \Gamma$. If $\chi \neq \gamma$, (5) implies that $\Psi_{A \chi} \neq \Psi_{B\}}$. If $A \neq B$ (say $A \not \supset B$ ), take any $\beta \in B \backslash A$; we claim

$$
M_{c}\left(L_{\beta}^{\prime}\right) \subset \operatorname{Ker} \Phi_{\ldots} \quad \text { where } \mathscr{T}=\mathscr{T}_{A} .
$$

In fact, let $T^{\prime}$ be an arbitrary subgroup in $\mathscr{T}$; there exists a countable subset $A_{0}$ of $A$ such that $T^{\prime}=G_{p}\left(\bigcup\left\{L_{n}^{\prime}: \alpha \in A_{0}\right\}\right)$. Since $K^{\prime}$ is independent and since $L_{\beta}^{\prime}$ and $\bigcup\left\{L_{\alpha}^{\prime}: \alpha \in A_{0}\right\}$ are disjoint subsets of $K^{\prime}$, it follows that $L_{\beta}^{\prime} \cap\left(x^{\prime}+T^{\prime}\right)$ contains at most one point for each $x^{\prime} \in G_{0}$. In particular, if $\mu^{\prime} \in M_{c}\left(L_{\beta}^{\prime}\right)$, then $\left|\mu^{\prime}\right|\left(x^{\prime}+T^{\prime}\right)=0$ for all $x^{\prime} \in G_{0}$. Since $T^{\prime} \in \mathscr{T}$ was arbitrary, we see that (7) holds. On the other hand, we have $\Phi_{M}\left(M\left(L_{\beta}^{\prime}\right)\right)=M\left(L_{\beta}^{\prime}\right)$ for $\mathscr{C}=\mathscr{T}_{B}$. Thus $\Psi_{A} \neq$ $\Psi_{B \gamma}$, as is easily seen. This clearly establishes Theorem 2.

REMARKs. ( i ) If $G$ is a metrizable $I$-group, then the element $x_{0}$ in Theorem 1 (and Corollary) can be chosen $x_{0}=0$. In fact, take any nonzero $\lambda_{0} \in M_{0}^{+}(G)$, and assume that $E_{q y}=\{x \in G: q x=y\}$ has positive $\lambda_{0}$-measure for some $q \in N$ and some $y \in G$. Let $\mu_{0}$ be the restriction of $\lambda_{0}$ to $E_{q y}$, so that $0 \neq \ell_{0}=M_{0}^{-}(G)$. It is trivial that $E_{q y}$ is a coset of some closed subgroup $H$ of $G$ which is of bounded order. If $\Gamma$ is the annihilator of $H$, we see $\left|\hat{\mu}_{0}\right|=$ const $\neq 0$ on $\Gamma$. Since $\hat{\mu}_{0}$ vanishes at infinity, it follows that $\Gamma$ is compact, or, equivalently, that $H$ is an open subgroup of $G$. This is a contradiction because $G$ is an $I$-group while $H$ is of bounded order. Thus, our assertion follows from the last paragraph of the proof of Lemma 2 and the proof of Theorem 1 for metrizable groups.

(ii) Let $\hat{G}^{-}$denote the closure of $\hat{G}$ in the maximal ideal space $\Delta_{G}$ of $M(G)$, and let $Y$ be as in Theorem 2. Then, for some $\tau \in M_{0}(G)$, the set $E_{\text {; }}$ of all symmetric $\Theta \in \Delta_{G}$ such that

$$
\bigcup\left\{L^{1}(\mu): \mu \in Y\right\} \cup M_{a}(G) \subset \Theta \text { and } \hat{\tau}(\Theta)=1
$$

has cardinal number $\geqq 2^{\omega}$, where $\hat{\tau}$ denotes the Gelfand transform of $\tau$. Note that $E_{\tau}$ is a closed subset of $\Delta_{G}$ disjoint from $\hat{G}^{-}$. To see this, redefine $\mathscr{P}(W)$ in the proof of Theorem 2 to be the family of all subsets of $W$ containing $1 \in W$, and fix any probability measure $\tau \in M_{0}^{+}(G)$ such that $\tau^{\prime} \in M\left(L_{1}^{\prime}\right)$. Then we have 


$$
\Psi_{A 1}(\tau)=\hat{\tau}^{\prime}(1)=\hat{\tau}(1)=1 \quad(A \in \mathscr{P}(W)) .
$$

(iii) Let $Y$ be as in Theorem 2. Then there exist a measure $\tau \in M_{0}^{-}(G)$, a nondiscrete LCA group $G_{0}$, and an independent compact subset $K^{\prime}$ thereof, with $M_{0}\left(K^{\prime}\right) \neq\{0\}$, having the following property: the set of all asymmetric $\Theta \in \Delta_{G}$ such that

$$
\bigcup\left\{L^{1}(\mu): \mu \in Y\right\} \cup M_{a}(G) \subset \Theta \text { and } \hat{\tau}(\Theta)=1
$$

has cardinal number $\geqq$ Card $M_{c}\left(K^{\prime}\right)^{*}$, where $M_{c}\left(K^{\prime}\right)^{*}$ denotes the conjugate space of $M_{c}\left(K^{\prime}\right)$. This can be proved using the proof of Theorem 2 and a theorem of Hewitt and Kakutani [2]. We omit the details.

(iv) Some analogs to our results hold for non-abelian groups. For example, we have the following: Let $G$ be a nondiscrete locally compact group, $\left\{\lambda_{k}\right\}_{k=0}^{\infty} \subset M_{c}^{+}(G), \lambda_{0} \neq 0$, and let $\left\{C_{k}\right\}_{i=0}^{\infty}$ be a countable family of $\sigma$-compact subsets of $G$ such that

$$
\lambda_{k}\left(x C_{k}\right)=0 \quad(x \in G ; k=0,1,2, \cdots) .
$$

Then there exists a nonzero measure $\sigma \in M_{c}^{+}\left(\operatorname{supp} \lambda_{0}\right)$ with compact support such that

$$
\lambda_{i:[}\left[x G_{p}(\operatorname{supp} \sigma) C_{k}\right]=0 \quad(x \in G ; k=0,1,2, \cdots) .
$$

\section{REFERENCES}

1. E. Hewitt, The asymmetry of cerain algebras of Fourier-Stielijes transforms, Michigan Math. J., 5 (1955), 149-158.

2. E. Hewitt and S. Kakutani, A class of multiplicative linear functionals on the measure algebra of a locally compact abelian group, Illinois J. Nath., 4 (1960), 553-574. 3. E. Hewitt and K.A. Ross, Absìract Harmonic Analysis, Vol. 1, Springer-Verlag, Heidelberg, 1963.

4. E. Hewitt and K.R. Stromberg, A remark on Fourier-Stieltjes transforms, An. Acad. Brasil. Ci., 34 (1962), 175-180.

5. L.-A. Lindahl and F. Pculsen, Thin Sets in Harmonic Analysis, Marcel-Dekker, Inc., New York, 1971.

6. L. Pigno and S. Saeki, Fourier-Siieltjes transforms which vanish at infinity, (submitted).

7. W. Rudin, Fourier-Stieltjes iransforms of measures on indeperdent sets, Bull. Amer. Math. Soc., 66 (1960), 199-202.

8. - Fourier Analysis on Groups, Interscience, New York, 1962.

9. N. Th. Varopoulos, Sets of multiplicity in locally compact abelian groups, Ann. Inst. Fourier (Grenoble), 16 (1966), 123-158.

Received March 15, 1973 and in revised form Jure 22, 1973.

Kansas State University

AND

Tokyo Metropolitan University 



\section{PACIFIC JOURNAL OF MATHEMATICS}

\section{EDITORS}

RICHARD ARENS (Managing Editor)

University of California

Los Angeles, California 90024

R. A. Beaumont

University of Washington

Seattle, Washington 98105
J. DugundJI

Department of Mathematics University of Southern California Los Angeles, California 90007

D. Gilbarg AND J. Milgram Stanford University

Stanford, California 94305

\section{ASSOCIATE EDITORS}
E. F. BECKENBACH
B. H. NeUmanN
F. WOLF
K. YOSHIDA

\section{SUPPORTING INSTITUTIONS}

UNIVERSITY OF BRITISH COLUMBIA CALIFORNIA INSTITUTE OF TECHNOLOGY

UNIVERSITY OF CALIFORNIA

MONTANA STATE UNIVERSITY

UNIVERSITY OF NEVADA

NEW MEXICO STATE UNIVERSITY

OREGON STATE UNIVERSITY

UNIVERSITY OF OREGON

OSAKA UNIVERSITY
UNIVERSITY OF SOUTHERN CALIFOF

STANFORD UNIVERSITY

UNIVERSITY OF TOKYO

UNIVERSITY OF UTAH

WASHINGTON STATE UNIVERSITY UNIVERSITY OF WASHINGTON

AMERICAN MATHEMATICAL SOCIET NAVAL WEAPONS CENTER 


\section{Pacific Journal of Mathematics}

\section{Vol. 54, No. 1 \\ May, 1974}

Ralph K Amayo, Engel Lie rings with chain conditions ..................

Bernd Anger and Jörn Lembcke, Hahn-Banach type theorems for hypolinear

functionals on preordered topological vector spaces ..................

Gregory Frank Bachelis and Samuel Ebenstein, On $\Lambda(p)$ sets ................

Harvey Isaac Blau, Indecomposable modules for direct products of finite

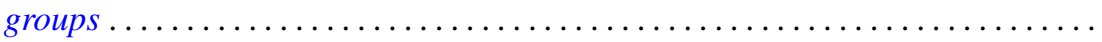

Larry Eugene Bobisud and James Calvert, Singular perturbation of a

time-dependent Cauchy problem in a Hilbert space ................

Walter D. Burgess and Robert Raphael, Abian's order relation and orthogonal

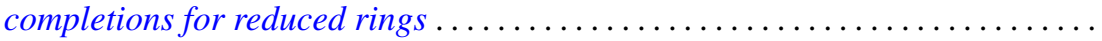

James Diederich, Representation of superharmonic functions mean continuous at

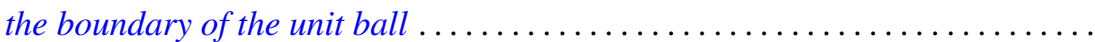

Aad Dijksma and Hendrik S. V. de Snoo, Self-adjoint extensions of symmetric

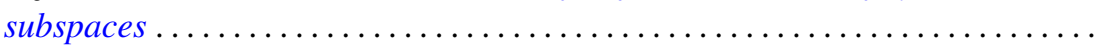

Gustave Adam Efroymson, A Nullstellensatz for Nash rings . . . . . . . . . . . . .

John D. Elwin and Donald R. Short, Branched immersions onto compact orientable surfaces . . . . . . . . . . . . . . . . . . . . . . . . .

John Douglas Faires, Comparison of the states of closed linear

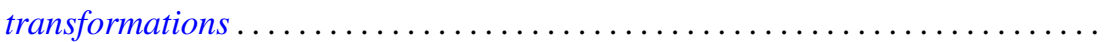

Joe Wayne Fisher and Robert L. Snider, On the von Neumann regularity of rings with regular prime factor rings .

Franklin Takashi Iha, A unified approach to boundary value problems on compact intervals

Palaniappan L. Kannappan and Che Tat $\mathrm{Ng}$, On functional equations connected with directed divergence, inaccuracy and generalized directed divergence

Samir A. Khabbaz and Elias Hanna Toubassi, The module structure of Ext $(F, T)$ over the endomorphism ring of $T$...

Garo K. Kiremidjian, On deformations of complex compact manifolds with boundary.

Dimitri Koutroufiotis, Mappings by parallel normals preserving principal

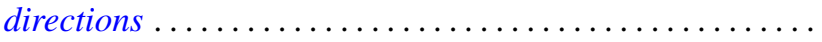

W. K. Nicholson, Semiperfect rings with abelian adjoint group

Norman R. Reilly, Extension of congruences and homomorphisms to translational hulls

Sadahiro Saeki, Symmetric maximal ideals in $M(G)$

Brian Kirkwood Schmidt, On the homotopy invariance of certain functors ...

H. J. Shyr and T. M. Viswanathan, On the radicals of lattice-ordered rings ...

Indranand Sinha, Certain representations of infinite group algebras ...

David Smallen, The group of self-equivalences of certain complexes ...

Kalathoor Varadarajan, On a certain problem of realization in homotopy

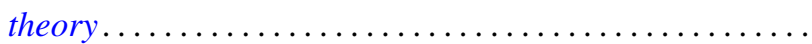

